\title{
Report on the Tenerife Workshop on Uncertainties in Atomic Data and How They Propagate in Chemical Abundances
}

\author{
Valentina Luridiana and Jorge García-Rojas \\ Instituto de Astrofísica de Canarias, \\ Vía Láctea s/n, La Laguna, E-38205, Spain, and \\ Departamento de Astrofísica, Universidad de La Laguna, \\ La Laguna, E-38205, Spain \\ email: vale, jogarcia@iac.es
}

\begin{abstract}
In 2010, we organised a workshop in Tenerife with the aim of bringing together scientists concerned with the completeness and accuracy of atomic data for astrophysical applications. Participants included atomic physicists, theoretical astrophysicists and astronomers, and the workshop covered topics such as the evaluation of uncertainties in atomic data, the propagation of such uncertainties in chemical abundances, and the feedback between observations and calculations. Communication issues were also discussed, including questions such as: How can it be ensured that atomic data are correctly understood and used? Which forum is the best one for a fluid interaction between all communities involved in the production and use of atomic data? This contribution lays out the main issues raised during the workshop and some of the solutions proposed.
\end{abstract}

Keywords. Atomic data, ISM: abundances, stars: abundances

\section{Introduction}

Atomic data, such as energy levels, radiative transition probabilities and collisional excitation rates, are a necessary ingredient in the modelling of astrophysical plasmas. When choosing which data to use for a particular calculation, astrophysicists need to assess which particular dataset is the most reliable and decide how to fill in gaps in selected datasets. Additionally, they often wish to assess the uncertainties in the chosen data in order to know how they will affect the accuracy of their final results. These are formidable tasks for those lacking a deep knowledge of atomic physics, and yet results may depend strongly on such choices. The workshop Uncertainties in Atomic Data and How They Propagate in Chemical Abundances, held in Tenerife (Spain) during 25-27 October 2010, was devoted to explore these issues and their solutions. In this paper we report on the discussions held during the workshop and the main conclusions that emerged from such discussions. Since many of these issues can be mitigated, if not solved, by a more fluid communication between experts in the various fields involved, the workshop wrapped up with a commitment to put in place a mechanism to favor such communication.

\section{Do uncertainties matter?}

The fundamental topic addressed by the workshop is summarized by a very simple question: do uncertainties matter? Since a picture tells more than one thousand words, there is no better answer to such question than a plot describing a real science case. Fig. 1 (courtesy of M. Nũnez-Díaz) represents the physical conditions of a Herbig-Haro 
object in Orion determined with several combinations of published [S II] atomic data (Podobedova et al. (2009); Ramsbottom et al. (1996); Tayal \& Zatsarinny (2010)). It can easily be seen that the results depend strongly on the data used: for example, the peak density changes by up to a factor of 3 (from $\approx 5000 \mathrm{~cm}^{-3}$ to $\approx 15000 \mathrm{~cm}^{-3}$ ), while the peak temperature changes by $\approx 5000 \mathrm{~K}$.

In most cases, we might not be aware of the existence of such dependency, but the truth is that many important astrophysical questions, including the synthesis of primordial He, stellar evolution models, stellar atmosphere models, yields for supernovae of various types, etc., depend upon accurate atomic data. More directly, the interpretation of comparisons between theory and observations depends critically on thorough assessments of the uncertainties in chemical abundances that are derived from atomic data. Accurately quantifying the effect of uncertainties is often difficult because of the complex ways in which different types of atomic data affect the calculations. For example, in computing elemental abundances from emission lines in a spectrum one has, on the one hand, the effects of uncertainties from collisional excitation rates and transitions probabilities on the derived ionic abundances, and, on the other, the effects of photoionization and recombination rates on the ionization correction factors.

In addressing this problem, it is crucial to count with the feedback of all people involved: atomic data producers (atomic physicists), users (astrophysicists who make calculations that depend on the data), and assessors (providers of software or tools that depend on the choice of atomic data). Representatives of these three categories were invited to the workshop.

\section{Articulating the problem}

The basic question we started from - do uncertainties in atomic data matter? - can be articulated in a set of more specific questions, which are listed below together with a summary of the discussion that followed. A more complete account can be found in
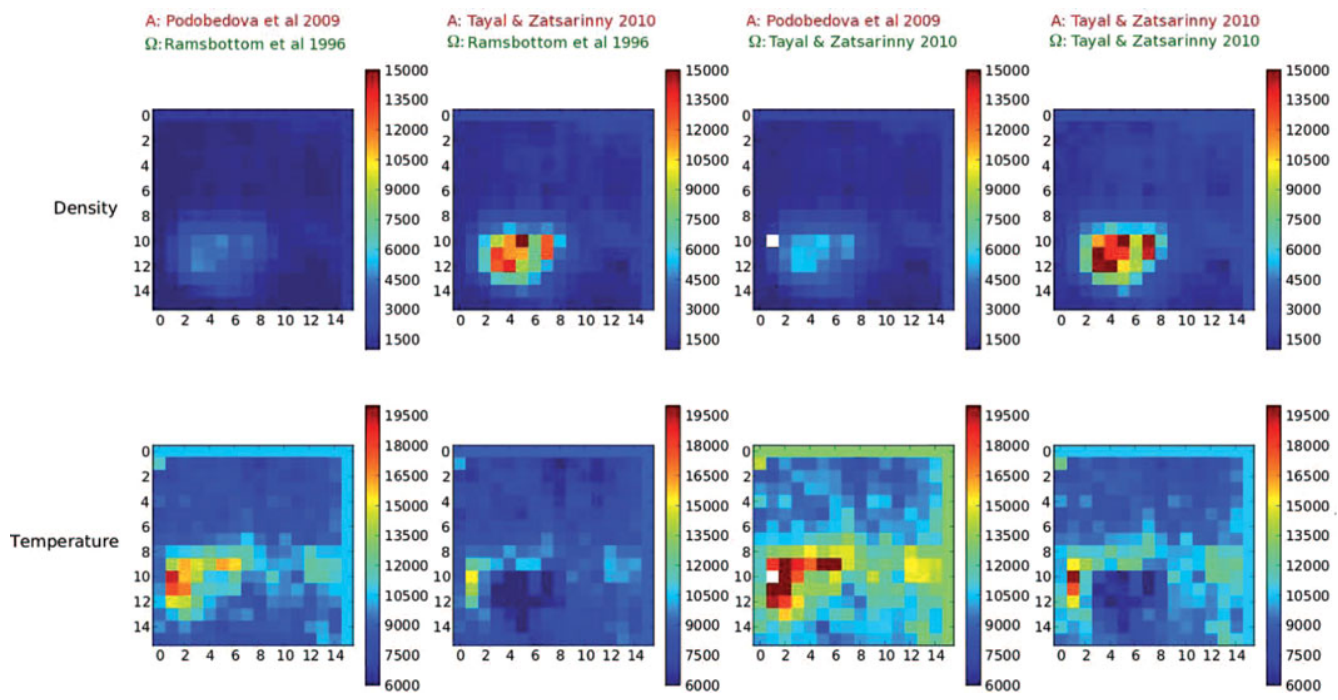

Figure 1. Physical conditions in the Herbig-Haro object HH204, computed with different combinations of atomic data. Upper row: $\left[\begin{array}{ll}\mathrm{S} & \mathrm{II}\end{array}\right]$ electronic density. Bottow row: $[\mathrm{S}$ II] electronic temperature. The observational data were obtained with PMAS at CAHA and are a courtesy of M. Núñez Díaz. 
Luridiana et al. (2011) and the complete proceedings can be downloaded from the website http://astroatom.files.wordpress.com/2011/10/atom2010.pdf.

\subsection{How Large Are Typical Uncertainties?}

The answer to this question crucially depends on the particular calculations and its scope. Not all the ions can be modeled equally well, and not all the ranges in temperature are equivalent; processes that are negligible in one domain may dominate in other domains, in such a way that the approximations used affect differently different ranges. Generally speaking, strong transitions are better known than weak transitions; energy levels are less uncertain than transition probabilities, which are in turn less uncertain than collision strengths; near thresholds, collision strengths are dominated by resonances, and the difficulty in pinning down the exact position and features of these may affect the accuracy of calculations, so that high temperature regimes are often better modeled than low temperature regimes.

\subsection{Why are uncertainties not usually specified?}

Ideally, atomic data should be provided with a quantitative estimate of the accuracy associated with them. In practice, this is almost never done, for multiple reasons that boil down to a simple issue: uncertainties in atomic calculations are very difficult to estimate. One reason for this is that benchmarking theoretical calculations -the source of most atomic data- against experimental values requires an enormous effort in term of human time and equipment, so it is not usually done; as a consequence, a proper assessment of the models adopted cannot be done.

\subsection{How can a user of atomic data estimate such uncertainties and decide which datasets to use?}

Since there are no standardized ways to quantify uncertainties on atomic data, users have to make their own assessment of the accuracy of the data they use, in order for both choosing among alternative datasets and assessing the uncertainties introduced by atomic data in their final results.

A surprisingly simple way to achieve this is by asking either the author or other specialists who might be able to give, if not a formal error estimate, at least an informed opinion on the data. Other users may also be helpful by providing information on which data they use, why, and whether they get consistent results with them. Indications of the accuracy of a given dataset can also be obtained by comparing it to other calculations, to laboratory measurements or to observational data (always remembering that consistency may suggest that the results have converged, but also that both calculations suffer from a common, unknown bias). Benchmarking against laboratory data is possible only for a handful of data, as explained above, so that energy levels are almost the only kind of data for which obtaining laboratory data is feasible. Measurements of collisional cross sections at a few energies may also be helpful for validating theory, but they may be intrinsically uncertain and suffer from the obvious limitation of not covering an entire energy range. Finally, theoretical data can be benchmarked against observations to provide estimates of uncertainties; real-life examples are the comparison between the observed and predicted [O III] 4959/5007 ratio, the [S II] temperature, or the intensities of the [Ne V] infrared lines in nebulae (observational errors on the line intensities must, of course, be taken into account). 


\section{Advice to researchers}

One important outcome of the workshop was the following advice list to researchers involved in the production and use of atomic data:

Advice to producers. Producers should always compare explicitly to previous data; in case of large differences, they should try to understand and explain them without making assumptions. The calculations should be tailored to the potential market.

Advice to assessors. Clear, explicit information on the datasets chosen and the reasons underlying the choice is vital to promote a responsible use of atomic data among users. Publicizing which data are used will also help producers obtaining those citations that are so crucial to getting funds.

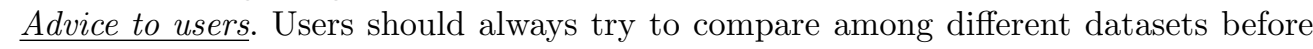
deciding for one. In case of doubts, asking the authors can often be a surprisingly effective way to understand features of the data. It should also be kept in mind that the newest data are not necessarily the best ones.

Users should also be aware of the range of validity of the data they are using and not extrapolate outside it. Finally, citing the source of the atomic data one is using is both a just reward for the authors and a way to ensure that the next piece of research will be funded.

\section{Communication within the field}

Fluent interaction between astrophysicists and atomic physicists has immediate benefits to all parties. It helps astrophysicists in deciding among different sources of atomic data and their uncertainties. It benefits producers of atomic data in helping them identify the most pressing user needs as well as opportunities for benchmarking the atomic data. We believe that this kind of communication between data users and producers is key to making the most of the existing data. To this aim, we decided to open a blog devoted to the discussion of atomic data and their astrophysical applications (http://astroatom.wordpress.com: see Fig. 2).

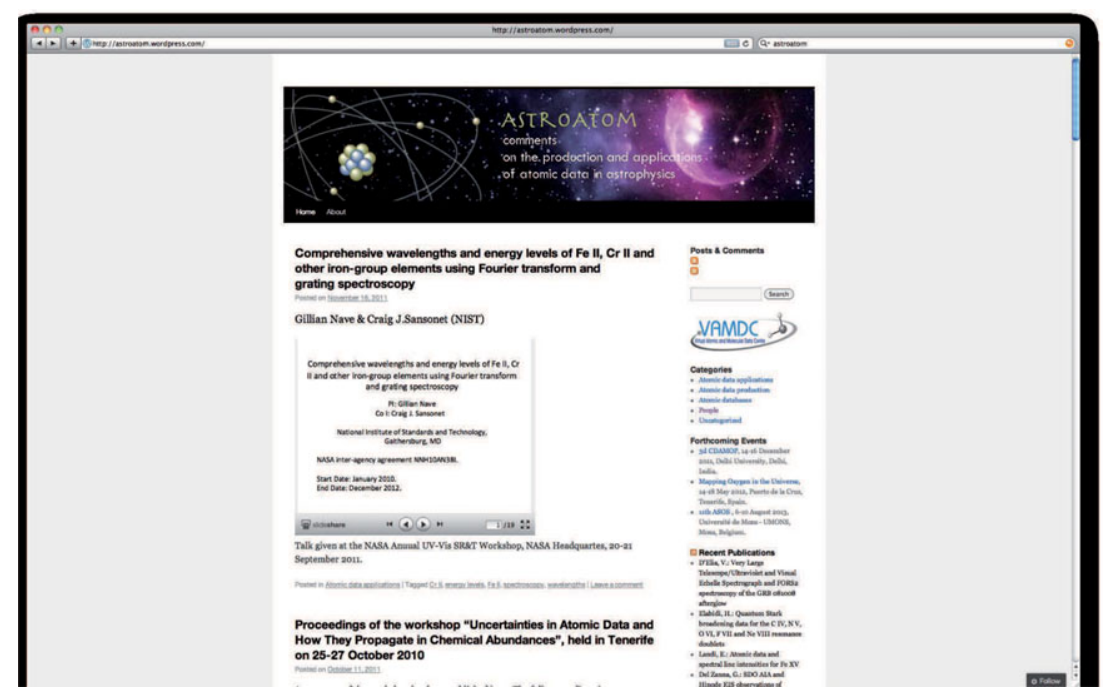

Figure 2. A snapshot of our new blog dedicated to the exchange of information on atomic data (http://astroatom.wordpress.com/). 
Our blog has quickly become both a communication channel and a repository of handy information on atomic data. If you are interested in participating, you are welcome to register and send your contribution.

\section{References}

Luridiana, V., García-Rojas, J. et al. 2011, arXiv:1110.1873

Podobedova, L. I., Kelleher, D. E., \& Wiese, W. L. 2009, J. Phys. Chem. Ref. Data, Vol. 38, No. 2,17

Ramsbottom C. A., Bell K. L., \& Stafford, R. P. 1996, ADNDT, 63, 57

Tayal, S. S. \& Zatsarinny, O. 2010, ApJS, 188, 32 